\section{Principal component analysis: A BASIC program for microcomputers}

\author{
DANIEL COULOMBE \\ University of Ottawa, Ottawa, Ontario, Canada
}

Principal component analysis can be viewed as a first step in factor analysis (Kleinbaum \& Kupper, 1978) or as a form of factor analysis (Laforge, 1981). Its purpose is to generate $\mathrm{K}$ orthogonal linear combinations from $\mathbf{P}$ $(\mathrm{P}>\mathrm{K})$ intercorrelated observed variables. The rationale underlying this technique is presented in Hotelling (1933) and in most textbooks on multivariate data analysis (Harris, 1975; Marascuilo \& Levin, 1983; Tabachnick \& Fidell, 1983). One interesting use of principal component analysis is that of providing a set of orthogonal variates that can be used in a multiple regression analysis. This procedure represents an elegant way of circumventing the problem of multicollinearity (Draper \& Smith, 1981).

The purpose of the program presented here is to perform principal component analysis, including orthogonal rotation of axes, using the varimax solution. Factorial scores computed from unrotated or rotated structure matrices can be generated and stored so that they can be input into the multiple regression system described in an earlier paper (Coulombe, 1983). All critical computations are performed in double precision to minimize rounding errors. The routines to obtain the eigenstructure of the correlation matrix, as well as the algorithm to obtain the varimax solution, were derived from Veldman's (1967) work.

Input. At the start of the program, the user is provided with a menu from which the data-input mode can be selected. Data (raw scores or correlation matrix) can be entered either from the keyboard or from a disk file. In either case, the program requests the number of subjects, the number of variables in the data set, and the minimum eigenvalue to extract. If raw scores are to be entered from the keyboard, the program prompts sequentially for each score obtained by a subject on each variable. If raw data are to be input from disk files, the program prompts for the name of each file to be read. If necessary, the data files can be created and edited with a data file editor described previously (Coulombe, 1983). If a correlation matrix is to be input from the keyboard, the program prompts for each upper triangular element of $\mathrm{R}$. Alternatively, if a correlation matrix is to be entered from a disk file, the program requests the name of the file containing the correlation matrix. Such a matrix can be created using a provided utility (CORMAT.BAS) from raw scores stored in disk files.

The author's mailing address is: School of Psychology, University of Ottawa, 275 Nicholas, Ottawa, Ontario, Canada K1N 6N5.
Output. All outputs are directed to the screen display. To obtain line-printer output, the PRINT statements should be changed to LPRINT statements or to any routing instructions available on the system in use. Output includes a listing of: (1) the means and standard deviations observed for each variable, when raw data are input; (2) the correlation matrix between all pairs of variables; (3) the determinant of R and Bartlett's test of sphericity; (4) the variances of each component and the percentage of trace; (5) the unrotated pattern matrix (i.e., principal component weights, loadings, or eigenvectors); (6) the distribution of variance of observed variables across principal components, in both actual and percentage form, and both uncumulated and cumulated across components; (7) the unrotated structure matrix (i.e., the correlations between the observed variables and the components); and (8) the varimax rotated structure and pattern matrices. Finally, if raw data were input, the user is provided with the possibility of computing factorial scores for any component, either in the unrotated or the rotated form. If those scores are computed, they are saved on disk files for ulterior use, in a format compatible with the MULREG system (Coulombe, 1983).

Program Language and Requirements. The two programs contained in the present system (PRINCOMP.BAS and CORMAT.BAS) were written in GW-BASIC on a 384K Tandy 2000 with two disk drives, under MS-DOS. This language is directly compatible with that of the IBM PC and work alikes. Both programs can be easily transported to other computers using Microsoft BASIC. The PRINCOMP.BAS program occupies about $9.0 \mathrm{~K}$ of memory, leaving approximately $52 \mathrm{~K}$ for execution. Correlation matrices including 45 variables can be input and analyzed on the Tandy 2000 and IBM PC. If raw data are input, problems involving 500 subjects measured on 20 variables can be analyzed. A larger data set can be analyzed by first computing the correlation matrix using CORMAT.BAS and then feeding this matrix to PRINCOMP.BAS.

The computation time required for an analysis depends on the computer clock speed, the number of subjects and variables, and the number of extracted factors. A problem involving 200 subjects and 20 variables was solved in less than $20 \mathrm{~min}$ on the Tandy 2000 (8-MHz clock speed). On that particular occasion, 10 factors were extracted.

Availability. A listing of the programs is available free of charge from the author, School of Psychology, University of Ottawa, 275 Nicholas, Ottawa, Ontario, Canada K1N 6N5.

\section{REFERENCES}

Coulomвe, D. (1983). MULREG: A multiple regression analysis system for microcomputers. Behavior Research Methods \& Instrumentation, 15, 608 .

DraPer, N., \& Smith, H. (1981). Applied regression analysis. New York: Wiley 
HaRris, R. J. (1975). A primer of multivariate statistics. New York: Academic Press.

Hotelling, H. (1933), Analysis of a complex of statistical variables into principal components. Journal of Educational Psychology, 24, 417-441, 498-520.

Kleinbaum, D. G., \& KUPPER, L. L. (1978). Applied regression analysis and other multivariable methods. North Scituate, MA: Duxbury Press.

LAFORGE, H. (1981). Analyse multivariée. Québec: Editions Etudes Vivantes.
Marascuilo, L. A., \& LeVIn, J. R. (1983). Multivariate statistics in the social sciences: A researcher's guide. Monterey, CA: Brooks/Cole.

TABAChNICK, B. G., \& FIDELL, L. S. (1983). Using multivariate statistics. New York: Harper \& Row.

VELDMAN, D. J. (1967). FORTRAN programming for the behavioral sciences. New York: Holt, Rinehart, \& Winston.

(Revision accepted for publication September 19, 1984.) 